\title{
From sciatica pain to a complex diagnosis: a case of pyomyositis and chronic lymphocytic leukemia
}

\author{
Ottavia Magnani, Matteo Caiti, Elisabetta Cenni, Paolo Moscatelli \\ U.O. Medicina d’Urgenza, Ospedale Policlinico San Martino, Genova, Italy
}

\begin{abstract}
We described a case of a 50-year-old man who presented severe low back and leg pain that quickly evolved in severe septic shock. Clinical workouts led us to diagnose a rare condition as pyomyositis because of the identification of Staphylococcus aureus septicemia and multiple muscle collections. Although this rare condition rarely occurs in temperate climates, its prevalence recently increased and is often associated with an immunocompromised condition. In this case, the investigation of an immunological impairment revealed an underlying chronic lymphocytic leukemia/small cell non-Hodgkin lymphoma.
\end{abstract}

\section{Case Report}

A 50-year-old Moroccan man was admitted to the AE department complaining of fever, severe low back and leg pain for a week. He had already been in another hospital for the same symptoms and was discharged with a sciatic diagnosis. On past medical history, the patient reported a bilateral hips replacement 15 years before, following a car accident, and an infection at the right prosthesis six years later that was treated with antibiotic therapy. He presented a positive Lasegue sign and muscular weakness on both upper and lower limbs on clinical examination. Cervical and lumbar CT scans excluded signs of trauma or severe

Correspondence: Ottavia Magnani, U.O. Medicina d'Urgenza, Ospedale Policlinico San Martino, Largo Rosanna Benzi 10, 16132, Genova, Italy.

E-mail: ottavia.magnani@gmail.com

Key words: Pyomyositis; chronic lymphocytic leukemia; Staphylococcus aureus septicemia.

Contributions: all authors contributed to the final manuscript.

Conflict of interests: the authors declare no potential conflict of interests.

Informed consent: the authors obtained informed consent from the patient.

Received for publication: 7 October 2021.

Accepted for publication: 9 February 2021.

This work is licensed under a Creative Commons Attribution NonCommercial 4.0 License (CC BY-NC 4.0).

${ }^{\circ}$ Copyright: the Author(s), 2021

Licensee PAGEPress, Italy

Italian Journal of Medicine 2021; 15:179-183

doi:10.4081/itjm.2021.1386 discs protrusion. He received analgesic treatment with NSAID and steroids with no improvement. Because of the presence of abdomen distension and rebound tenderness on clinical examination, a CT scan was performed and revealed the presence of splenomegaly, hepatic and celiac lymph nodes, and substantial enlargement of bilateral iliac lymph nodes. Abnormal laboratory tests were WBC $17.44 \times 10^{9} / \mathrm{L}$ (neutrophil $13 \times 10^{9} / \mathrm{L}$ ), CRP $356 \mathrm{mg} / \mathrm{L}$, PLT 90,000/L, PCT 40.70 $\mu \mathrm{g} / \mathrm{L}, \mathrm{CPK} 2232 \mathrm{U} / \mathrm{L}$ increased serum creatinine $(1.3$ $\mathrm{mg} / \mathrm{L}$ ). Arterial blood gas analysis revealed high anion gap metabolic acidosis (pH: 7.323, $\mathrm{HCO}_{3}$ : $18.6 \mathrm{AG:}$ $13 \mathrm{mEq} / \mathrm{L})$ with increased lactate $(4.5 \mathrm{mmol} / \mathrm{L})$. Lung $\mathrm{X}$-ray showed the presence of lower-right hilar consolidations. He started i.v. hydration and antibiotic treatment with ceftriaxone, subsequently replaced with piperacillin + tazobactam. According to an infectious disease specialist, an extensive serological screening was performed to exclude HIV, listeria, pneumococcus, and legionella infections. Multiple blood cultures and QuantiFERON were performed. Because of the persistence of severe leg pain with difficulty to mobilization, a neurological evaluation was performed and revealed 4 limbs muscle weakness; spinal tap excluded Guillain Barré syndrome. Twentyfour hours after the admission, the patient presented a severe clinical deterioration with hypotension, the persistence of septic status, and increased creatinine serum level. He was moved to the intensive care department and started noradrenaline, oxygen supports, and intensive hydration. He continued a large spectrum antibiotic treatment with meropenem leading to a stabilization of the clinical status. He was transferred to the internal medicine department but suddenly presented a clinical worsening characterized by fever, tachypnoea, the appearance of limbs cellulitis with painful cramps and swollen areas. The patient presented anasarca with severe hypo-albuminemia status 
$(1.8 \mathrm{mg} / \mathrm{dL})$ and persistence of systemic and muscle inflammation laboratory signs. Meanwhile, hemoculture revealed Staphylococcus aureus, and accordingly, with an antibiogram, we started oxacillin i.v. QuantiFERON test revealed an undetermined result; Ig levels were normal. We performed a PET scan that revealed inflammatory areas localized in the anterolateral muscular compartment of the right tight and in the soft tissue near left sternum clavicular articulation (Figure $1)$; in the same contest, hypodense areas with no chemical activity were described, compatible with necrotic colliquative phenomena. Moderate collection of tracer was also observed in medial muscle areas of both arms, pectoral muscles. CT scan confirmed the presence of abscess muscle collection in both tights and at the iliopsoas, suggestive for pyomyositis (PM) (Figure 2). The patient underwent an emergency fasciotomy of the tight collections with vacuum-assisted closure and support treatment in the intensive care department (Figure 3).

After stabilization of the clinical conditions, he returned to our ward to complete the diagnostic approach and continue treatment. Despite the continuous intensive antibiotic treatment, we observed recurrence of muscle collections. Echocardiogram resulted negative for infective endocarditis. Cultures of fluid collection drainage from peri-prosthetic tissue excluded an infection of the prosthesis, whereas muscle biopsy demonstrated an unspecific inflammation pattern. The presence of an undetermined QuantiFERON result, the negative HIV test, and the exclusion of primary immunodepression suggested a functional impairment of the immune system or a secondary cause of immunodeficiency. Due to this suspicion, we performed a lymph node and a bone marrow biopsy, and a peripheral blood lymphocyte typing.

Lymph nodes biopsy revealed the loss of normal node architecture with diffuse atypical small lymphocyte proliferation and the presence of prolymphocytes and paraimmunoblasts. Neoplastic elements expressed CD20, CD79a, MUM-1, PAX-5, Bcl-2, CD-5 and $\mathrm{CD} 23$. Bone marrow biopsy was characterized by the presence of small cells lymphocytes infiltration with interstitial pattern and central medullary aggregation with analogous immunohistochemical expression. Lymphocyte typing confirmed the presence of the abnormal small size B cells population.

Histological and serological findings suggested evidence of chronic lymphocytic leukemia (CLL)/nonHodgkin small cells lymphoma. However, according to the hematology consultant, we decided to adopt a wait and see approach because of the patient's frailty and high risk of infection recurrence. After clinical improvement and a step-down antibiotic cycle with amoxicillin, the patient was referred to a hematology outpatient clinic and discharged with a diagnosis of septic status in pyomyositis and chronic lymphocytic leukemia.

\section{Discussion}

$\mathrm{PM}$ is a rare disorder characterized by the presence of muscle localization of infection. The infection, commonly caused by $S$. aureus, mainly involves muscles of lower limbs as quadriceps, iliopsoas followed by the gluteal muscle. The localization in the upper extremities and cervical musculature is less frequent. Several other pathogens than Staphylococcus have been described as associated with PM, including other Gramm positive or negative Bacteria and, even if less frequent, parasites and viruses. PM could also be re-
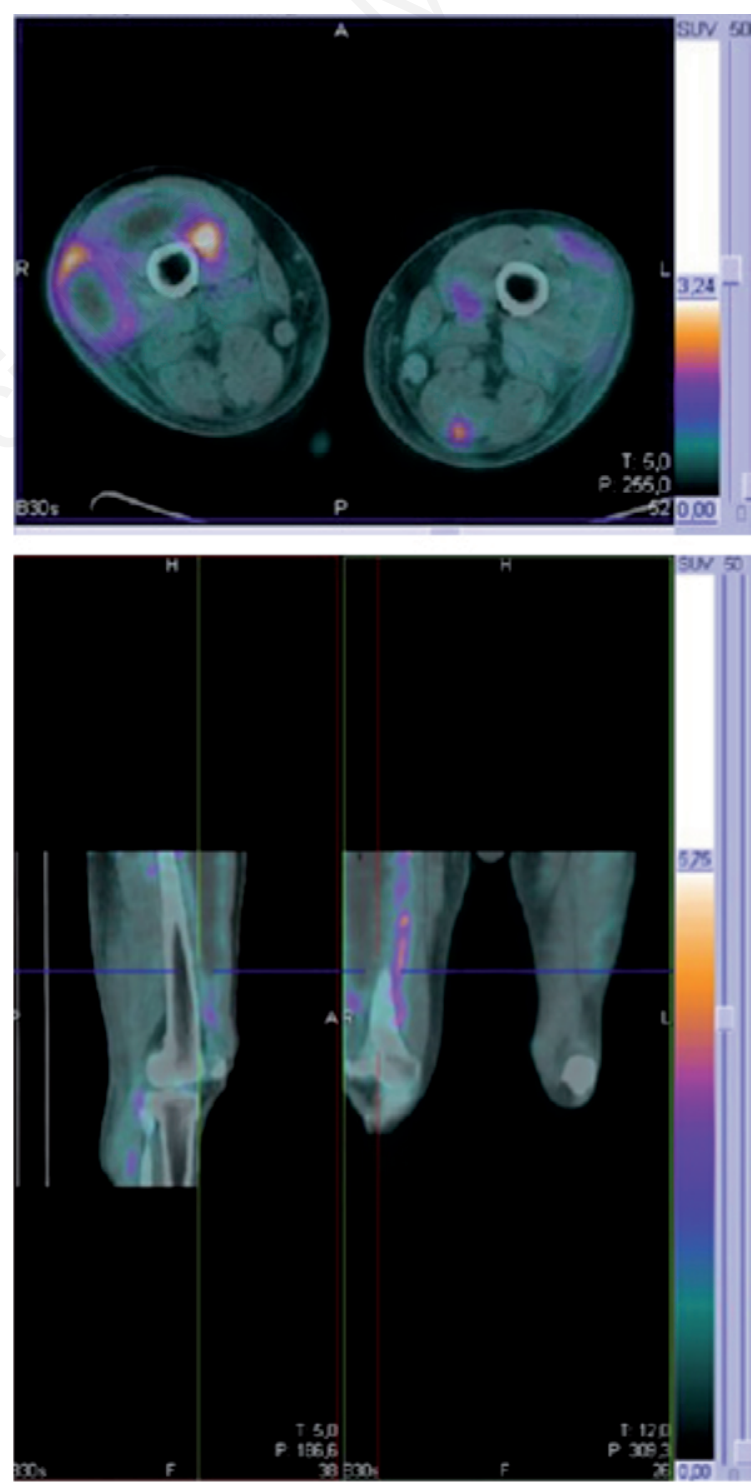

Figure 1. Positron emission tomography scan describing increased metabolism in thigh areas suggestive of inflammation. 
lated to factors affecting the muscle as strenuous exercise and direct muscle trauma. ${ }^{1,2}$ Clinically, it is possible to recognize different stages of the disease: in the first stage, the disease is primary localized in muscles with locoregional symptoms as swelling, tenderness, erythema; progressively, the disease could evolve in a suppurative phase followed by a systemic septic stage with severe manifestations. ${ }^{3}$

While the majority of cases are described in tropical areas where the disease is endemic, data about PM in temperate climates are spreading in literature. ${ }^{1,4} \mathrm{Al}$ - though the clinical characteristics of PM are similar, in tropical areas, the disease affects primarily young people and children and is associated with malnutrition. The underlying predisposing condition is an immunological impairment that facilitates the infection's spreading. In this contest, HIV infection could play a crucial role; ${ }^{5}$ the low CD4 count, the defect of phagocytosis, the retrovirus itself, and immunological impairment could facilitate the spreading of infection in seropositive patients. ${ }^{6}$ Otherwise, also other described causes of secondary immunodeficiencies as risk fac-

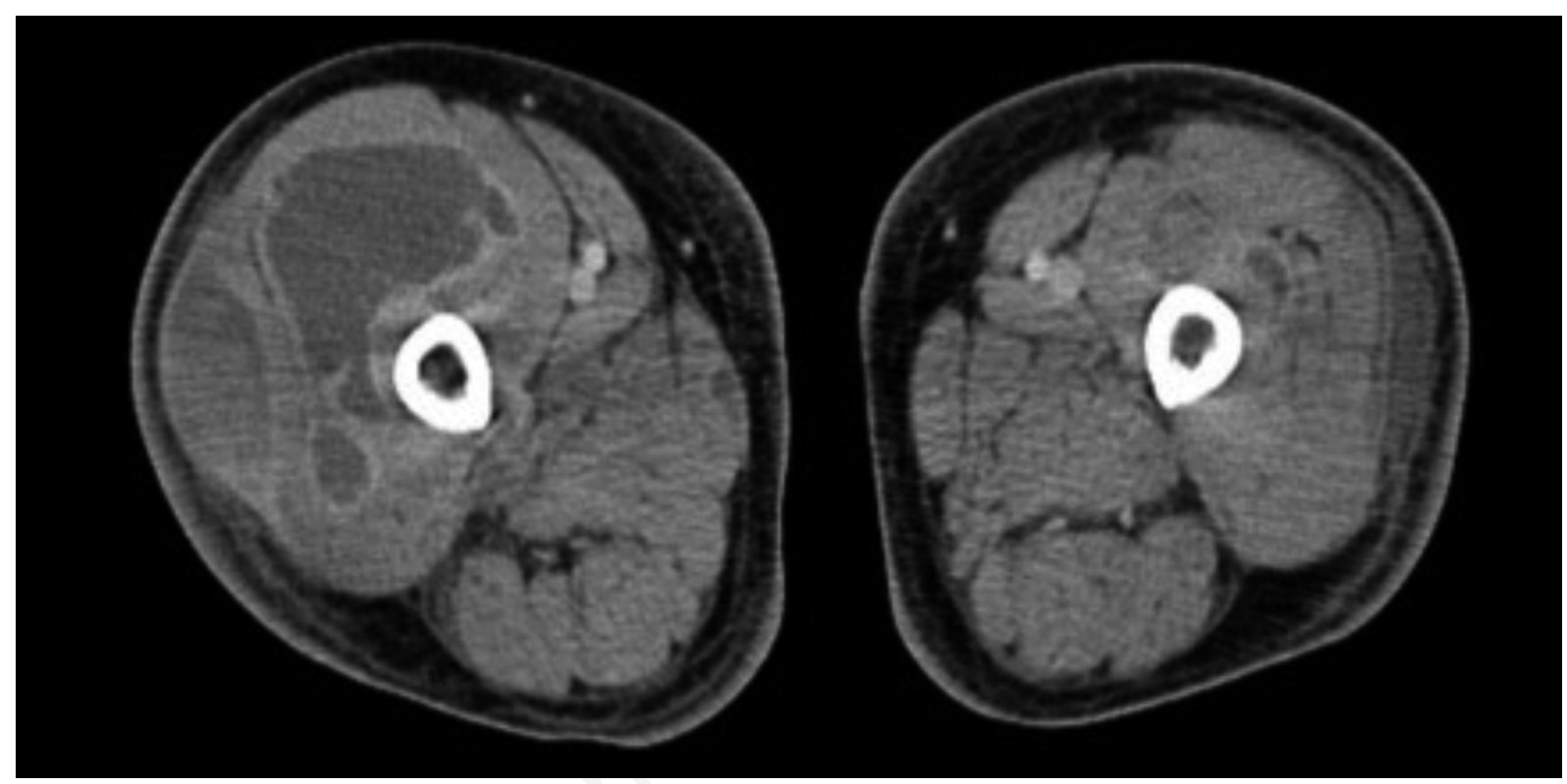

Figure 2. Computed tomographic scan revealed the presence of bilateral thigh intramuscular collections.

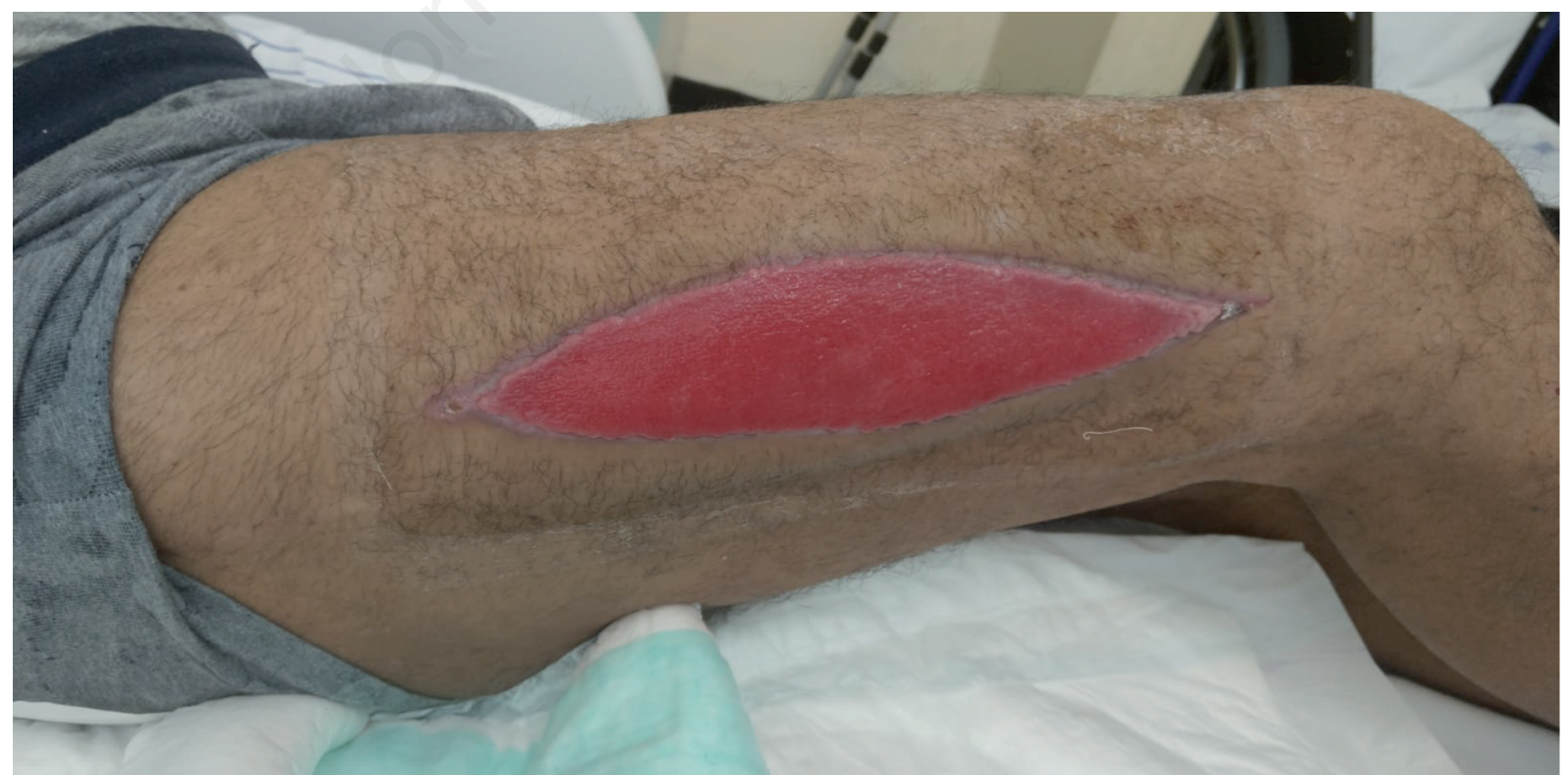

Figure 3. Result of surgical fasciotomy of the right tight. 
tors for PM are chronic use of steroids or administration of immunosuppressive drugs, diabetes, malignancy (especially hematologic), cirrhosis, renal insufficiency, and organ transplantation. ${ }^{7,8}$

According to literature data, early PM diagnosis is often missing because of the lack of familiarity with this disease. The pain was the leading symptom complained by our patient; as a matter of fact, he evolved to septic shock in almost 24 hours from the admission to the emergency department and required intensive care as the first approach.

Management of PM required the early institution of antibiotic treatment and also surgical debridement and drainage. Our patient switched empirically several drugs before blood culture results and the Staphylococcus identification, delaying a target therapeutic approach. The differential diagnosis is supported by imaging as CT MRI and ultrasound with the identification of muscle collection defining its extension and localization and the possibility of abscess drainage. In the described case, surgical intervention with the emergency fasciotomy allowed abscess drainage and prevented compartmental syndrome. Moreover, considering the patient medical history, we performed an accurate study of hip implant integrity and a drainage fluid collection from peri-prosthetic tissue to exclude a focus of the infection originating from prosthetic. The unspecific findings of muscle biopsy excluded other conditions as osteosarcoma or polymyositis.

In our report, the diagnosis of the underlying hematologic disorder was challenging and delayed because of the severity of clinical presentation and the rapidity of worsening of the condition.

Regarding the association between PM and hematological disorder, literature experiences suggest the importance of excluding an immunocompromised state, as the infection could be the unique manifestation of a hematological disorder. ${ }^{9-11}$

Undetermined QuantiFERON test, the enlarged lymph nodes, and the recurrence of infection despite the antibiotic treatment suggested an underlying immunological impairment and led us to more specific investigations. In addition, histological findings on laterocervical lymph node biopsy and bone marrow biopsy allowed the identification of a cellular neoplastic clone compatible with small cell non-Hodgkin's lymphoma/CLL.

CLL patients have an increased risk of pathogen colonization and opportunistic infections, and this condition represents a complication beyond a demonstrated hypogammaglobulinemia or concurrent immunosuppressive treatment. As described in the literature, the impairment of the classic immune response involves both innate and adaptive systems. A recent work demonstrated that monocyte in CLL patients presents a refractory state and a mechanism known as endotoxin tolerance, characterized by low proinflammatory cytokine production, elevated phagocytosis rate, low apoptosis frequency and impaired $\mathrm{Ag}$ presentation. ${ }^{12}$ In the reported situation, the immunological impairment secondary to the hematological disorder probably facilitated the spreading of the infection, complicated by muscle localization and septic shock. Although rare, it is important to be aware of PM, which represents a challenging disease to diagnose on physical examination but could be potentially life-threatening if missed. The continuous collaboration between intensive care and the internal medicine department was decisive for improving the medical condition of our patient and allowed an in-depth diagnostic course after stabilizing a critical condition. In this case, the multidisciplinary approach and the investigation of the immunological profile were crucial to identify the underlying hematological condition.

\section{Conclusions}

PM represents a rare but potentially severe condition because of the challenging diagnosis and lifethreatening complications often requiring combined medical and surgical intervention. Consequently, signs of inflammation and unspecific symptoms as pain and fever in an emergency department must be monitored to avoid a clinical aggravation as a septic status that may require intensive care. Nevertheless, the spreading of potentially immunocompromising conditions such as immunosuppressive treatments, transplants, and chronic diseases represents a risk factor for severe infections in general; the exclusion of secondary causes of immune impairment has to be carried out especially in the management of young patients presenting with severe septic status.

\section{References}

1. Comegna L, Guidone PI, Prezioso G, et al. Pyomyositis is not only a tropical pathology: a case series. J Med Case Rep 2016;10:1-6.

2. González Gómez B, Vargas Pérez M, del Rosal Rabes T, et al. Pyomyositis in a non-tropical area. 12 years of cased-based experience. An Pediatría (English Ed.) 2017;87:350-1.

3. Manikandan V, Mehrotra S, Anand S, Maurya V. Tropical pyomyositis: revisited. Indian J Surg 2017;79:33-7.

4. Gibson RK, Rosenthal SJ, Lukert BP. Pyomyositis. Increasing recognition in temperate climates. Am J Med 1984;77:768-72.

5. Christin L, Sarosi GA. Pyomyositis in North America: case reports and review. Clin Infect Dis. 1992;15:668-77.

6. Chauhan S, Jain S, Varma S, Chauhan SS. Tropical pyomyositis (myositis tropicans): current perspective. Postgrad Med J. 2004;80:267-70.

7. Charitidis C, Stampolidis N, Falidas E, Tsochataridis E. 
Multifocal pyomyositis and meningitis after bone marrow biopsy in a diabetic patient. G Chir. 2011;32:185-7.

8. Xipell M, Ventura-Aguiar P, Revuelta I, et al. Pyomyositis in a patient with IgA nephropathy and kidney transplant. Case Rep Transplant 2019;2019:1-3.

9. Takebayashi K, Sonoda H, Shimizu T, et al. Pyomyositis at the surgical site in a patient with chronic myeloid leukemia: a case report and literature review. World J Surg Oncol 2016;14:116.

10. Falagas ME, Rafailidis PI, Kapaskelis A, Peppas G. Py- omyositis associated with hematological malignancy: case report and review of the literature. Int J Infect Dis 2008;12:120-125.

11. Navinan MR, Yudhisdran J, Kandeepan T, Kulatunga A. Tropical pyomyositis as a presenting feature of subclinical leukemia: a case report. J Med Case Rep 2015;9.

12. Jurado-Camino T, Córdoba R, Esteban-Burgos L, et al. Chronic Lymphocytic leukemia: a paradigm of innate immune cross-tolerance. J Immunol 2014;194:719-27. 